1 Hacettepe Journal of Mathematics and Statistics

holume 44 (5) (2015), 1033-1044

\title{
Suborbital graphs for the group $\Gamma^{2}$
}

\author{
Bahadır Özgür Güler*†, Murat Beşenk ${ }^{\ddagger}$, Yavuz Kesicioğlu ${ }^{\S}$, Ali Hikmet Değer ${ }^{\natural}$
}

\begin{abstract}
In this paper, we investigate suborbital graphs formed by the action of $\Gamma^{2}$ which is the group generated by the second powers of the elements of the modular group $\Gamma$ on $\hat{\mathbb{Q}}$. Firstly, conditions for being an edge, self-paired and paired graphs are provided, then we give necessary and sufficient conditions for the suborbital graphs to contain a circuit and to be a forest. Finally, we examine the connectivity of the subgraph $F_{u, N}$ and show that it is connected if and only if $N \leq 2$.
\end{abstract}

Keywords: Modular group, Group action, Suborbital graphs

2000 AMS Classification: 20H05, 05C25

Received 13/11/2013 : Accepted 27/05/2014 Doi : 10.15672/HJMS.2015449656

\section{Introduction}

Let $\operatorname{PSL}(2, \mathbb{R})$ denote the group of all linear fractional transformations

$$
T: z \rightarrow \frac{a z+b}{c z+d}, \text { where } a, b, c \text { and } d \text { are real and } a d-b c=1 .
$$

In terms of matrix representation, the elements of $\operatorname{PSL}(2, \mathbb{R})$ correspond to the matrices

$$
\pm\left(\begin{array}{ll}
a & b \\
c & d
\end{array}\right) ; \quad a, b, c, d \in \mathbb{R} \text { and } a d-b c=1
$$

This is the automorphism group of the upper half plane $\mathbb{H}:=\{z \in \mathbb{C}: \operatorname{Im}(\mathrm{z})>0\}$.

The modular group $\Gamma=\operatorname{PSL}(2, \mathbb{Z})$, is the subgroup of $\operatorname{PSL}(2, \mathbb{R})$ such that $a, b, c$ and $d$ are integers. It is generated by the matrices

$$
U=\left(\begin{array}{cc}
0 & -1 \\
1 & 0
\end{array}\right) \quad ; \quad V=\left(\begin{array}{cc}
0 & -1 \\
1 & 1
\end{array}\right)
$$

\footnotetext{
*Dept. of Math., Karadeniz Tech. Uni., Turkey, Email: boguler@ktu.edu.tr

${ }^{\dagger}$ Corresponding Author.

${ }^{\ddagger}$ Dept. of Math., Karadeniz Tech. Uni., Turkey, Email: mbesenk@ktu.edu.tr

${ }^{\S}$ Dept. of Math., Recep Tayyip Erdogan Uni., Turkey, Email: yavuzkesicioglu@yahoo.com

"Dept. of Math., Karadeniz Tech. Uni., Turkey, Email: ahikmetd@ktu.edu.tr
} 
with defining relationships $U^{2}=V^{3}=I$, where $I$ is the identity matrix. $\Gamma$ is a Fuchsian group of signature $(0 ; 2,3, \infty)$, so it is isomorphic to a free product $C_{2} * C_{3}$.

Define $\Gamma^{m}$ as the subgroup of $\Gamma$ generated by the $m^{\text {th }}$ powers of all elements of $\Gamma$. Especially, $\Gamma^{2}$ and $\Gamma^{3}$ have been studied extensively by Newman $[13,14,15]$. It turns out that,

$$
\Gamma^{2}=\left\{\left(\begin{array}{ll}
a & b \\
c & d
\end{array}\right) \in \Gamma: a b+b c+c d \equiv 0(\bmod 2)\right\},
$$

by Rankin [Eq. 1.7.1, 16]. From the equation $a b+b c+c d \equiv 0(\bmod 2)$, we see that at least one of the integers $a, b, c, d$ must be even. Suppose first that $a=2 a_{0}$. Then using the determinant, we have that $b$ and $c$ are odd. So, $d$ must be odd as well. Hence, we get the elements of $\Gamma^{2}$ as the matrices $\left(\begin{array}{cc}2 a & b \\ c & d\end{array}\right)$. Similarly, supposing $d=2 d_{0}$, we can get the elements of the form $\left(\begin{array}{cc}a & b \\ c & 2 d\end{array}\right)$. Lastly, if $a$ or $d$ is not even, then both $b$ and $c$ will be even. To sum up, $\Gamma^{2}$ has three types of elements

$$
\left(\begin{array}{cc}
2 a & b \\
c & d
\end{array}\right),\left(\begin{array}{cc}
a & 2 b \\
2 c & d
\end{array}\right),\left(\begin{array}{cc}
a & b \\
c & 2 d
\end{array}\right)
$$

where $b, c$ and $d$ of the first, $a$ and $d$ of the second and $a, b, c$ of the third matrix are odd.

1.1. Theorem. [13] The group $\Gamma^{2}$ is the free product of two cyclic groups of order 3 , and

$$
\left|\Gamma: \Gamma^{2}\right|=2, \Gamma=\Gamma^{2}+\left(\begin{array}{cc}
0 & -1 \\
1 & 0
\end{array}\right) \Gamma^{2} .
$$

The elements of $\Gamma^{2}$ may be characterized by the requirement that the sum of the exponents of $\left(\begin{array}{cc}0 & -1 \\ 1 & 0\end{array}\right)$ be divisible by 2 .

The idea of a suborbital graph has been used mainly by finite group theorists. In [7], Jones, Singerman and Wicks showed that this idea is also useful in the study of the modular group, where they proved that the well-known Farey Graph is an example of a suborbital graph. Furthermore, they proved the following result:

Theorem A. The suborbital graph $G_{u, n}$ of $\Gamma$ contains directed triangles if and only if $u^{2} \pm u+1 \equiv 0(\bmod n)$.

Morever they posed the conjecture: $G_{u, n}$ is a forest if and only if it contains no triangles, that is, if and only if $u^{2} \pm u+1 \not \equiv 0(\bmod n)$. Akbas proved in [2] that this conjecture is true. By similar arguments, we concern with suborbital graphs of Picard group $\mathbf{P}$, which is the subgroup of $\operatorname{PSL}(2, \mathbb{C})$ with entries coming from $\mathbb{Z}[i]$ in [3]. Since $\mathbb{Z}[i]$ is a unique factorization domain with finitely many units, our expectation was to find similar formulas. Consequently, theorem A was improved as

Theorem B. The suborbital graph $G_{u, N}$ of $\mathbf{P}$ contains directed triangles if and only if $\varepsilon^{2} u^{2} \mp \varepsilon u \pm 1 \equiv 0(\bmod N)$.

In this study, we will continue to investigate the combinatorial properties of these graphs for the group $\Gamma^{2}$. It is an important subgroup of $\Gamma$ since all the groups $\Gamma^{m}$ can be expressed in the terms of $\Gamma, \Gamma^{2}, \Gamma^{3}$. The purpose of this paper is to characterize all circuits in the suborbital graph and connectedness for $\Gamma^{2}$. As it can be seen from Section 3 , we show that the main difference is in connectedness of related graphs. 


\section{The action of $\Gamma^{2}$ on $\hat{\mathbb{Q}}$}

Every element of $\hat{\mathbb{Q}}$ can be represented as a reduced fraction $\frac{x}{y}$ with $x, y \in \mathbb{Z}$ and $(x, y)=1$. This representation is not unique, because $\frac{x}{y}=\frac{-x}{-y}$. We represent $\infty$ as $\frac{1}{0}=\frac{-1}{0}$. The action of the matrix $\left(\begin{array}{ll}a & b \\ c & d\end{array}\right)$ on $\frac{x}{y}$ is

$$
\left(\begin{array}{ll}
a & b \\
c & d
\end{array}\right): \frac{x}{y} \longrightarrow \frac{a x+b y}{c x+d y}
$$

Hence, the actions of a matrix on $\frac{x}{y}$ and on $\frac{-x}{-y}$ are identical. If the determinant of the matrix $\left(\begin{array}{ll}a & b \\ c & d\end{array}\right)$ is 1 and $(x, y)=1$, then $(a x+b y, c x+d y)=1$.

\subsection{Transitive Action.}

2.1. Lemma. $\quad$ (i) The action of $\Gamma^{2}$ on $\hat{\mathbb{Q}}$ is transitive.

(ii) The stabilizer of a point is an infinite cyclic group.

Proof. (i) Here we only prove the case that any element of the form $\frac{a}{2 b}$ of $\hat{\mathbb{Q}}$ is sent $\infty$ by an element of $\Gamma^{2}$. The rest are similar. Let $\frac{a}{2 b} \in \hat{\mathbb{Q}},(a, 2 b)=1$. There exist integers $x_{0}$ and $y_{0}$ such that $a y_{0}-2 b x_{0}=1$ (known as Bezout's identity [8]). Hence, we have that $T:=\left(\begin{array}{cc}a & x_{0} \\ 2 b & y_{0}\end{array}\right) \in \Gamma$. All solutions of the equation $a y-2 b x=1$ are $x=x_{0}+a n$ , $y=y_{0}+2 b n$ for $n \in \mathbb{Z}$. If $x_{0}$ is odd, $x$ would be even by taking $n$-odd. So, $x_{0}$ can be chosen as an even number. Hence, $T \in \Gamma^{2}$ and $T(\infty)=\frac{a}{2 b}$ means that $\frac{a}{2 b}$ is in the orbit of $\infty$.

(ii) By (i), since the stabilizers of any two points in $\hat{\mathbb{Q}}$ are conjugate in $\Gamma^{2}$, it is sufficient to consider the stabilizer $\Gamma_{\infty}^{2}$ of $\infty$. It is clear that $\Gamma_{\infty}^{2}=\left\langle\left(\begin{array}{ll}1 & 2 \\ 0 & 1\end{array}\right)\right\rangle$.

We remark that Lemma 2.1 (i) can be proven by using the signature of $\Gamma^{2}$ as well. There is a homomorphism $\theta: \Gamma \longrightarrow C_{2}=\{e, \alpha\}$ defined by $\theta(U)=\alpha$, and $\theta(V)=e$. The kernel is $\Gamma^{2}$. By the permutation theorem [19], $\Gamma^{2}$ has signature $(0 ; 3,3, \infty)$. It means that there is only one orbit, so the action is transitive.

2.2. Imprimitive Action. We now discuss the imprimitivity of the action of $\Gamma^{2}$ on $\widehat{\mathbb{Q}}$. For this, let $(G, \Omega)$ be a transitive permutation group, consisting of a group $G$ acting on a set $\Omega$ transitively. An equivalence relation $\approx$ on $\Omega$ is called $G$-invariant if, whenever $\alpha, \beta \in \Omega$ satisfy $\alpha \approx \beta$, then $g(\alpha) \approx g(\beta)$ for all $g \in G$. The equivalence classes are called blocks. from

We call $(G, \Omega)$ imprimitive if $\Omega$ admits some $G$-invariant equivalence relation different

(i) the identity relation, $\alpha \approx \beta$ if and only if $\alpha=\beta$;

(ii) the universal relation, $\alpha \approx \beta$ for all $\alpha, \beta \in \Omega$.

Otherwise, $(G, \Omega)$ is called primitive. These two relations are supposed to be trivial relations.

2.2. Lemma. [4] Let $(G, \Omega)$ be a transitive permutation group. $(G, \Omega)$ is primitive if and only if $G_{\alpha}$, the stabilizer of $\alpha \in \Omega$, is a maximal subgroup of $G$ for each $\alpha \in \Omega$.

From the above lemma we see that whenever, for some $\alpha, G_{\alpha} \lesseqgtr H \lesseqgtr G$, then $\Omega$ admits some $G$-invariant equivalence relation other than the trivial one and the universal one. 
Because of the transitivity, every element of $\Omega$ has the form $g(\alpha)$ for some $g \in G$. Thus one of the non-trivial $G$-invariant equivalence relations on $\Omega$ by $H$ is given as follows:

$$
g(\alpha) \approx g^{\prime}(\alpha) \text { if and only if } g^{\prime} \in g H .
$$

The number of blocks (equivalence classes ) is the index $|G: H|$ and the block containing $\alpha$ is just the orbit $H(\alpha)$.

Let $N \in \mathbb{N}$ and let $\Gamma_{0}^{2}(N)$ be defined by

$$
\Gamma_{0}^{2}(N):=\left\{\left(\begin{array}{ll}
a & b \\
c & d
\end{array}\right) \in \Gamma^{2}: c \equiv 0(\bmod N)\right\}
$$

Then $\Gamma_{0}^{2}(N)$ is a subgroup of $\Gamma^{2}$. It is clear that $\Gamma_{\infty}^{2} \leq \Gamma_{0}^{2}(N) \leq \Gamma^{2}$ for $N \in \mathbb{N}$ and $\Gamma_{\infty}^{2} \lesseqgtr \Gamma_{0}^{2}(N) \lesseqgtr \Gamma^{2}$ for $N>1$.

2.3. Lemma. $\left|\Gamma_{0}(N): \Gamma_{0}^{2}(N)\right|=2$. In fact,

$$
\Gamma_{0}(N)=\left\{\begin{array}{ll}
\Gamma_{0}^{2}(N) \cup\left(\begin{array}{cc}
1 & 0 \\
N & 1
\end{array}\right) \Gamma_{0}^{2}(N), & N \text { is odd } \\
\Gamma_{0}^{2}(N) \cup\left(\begin{array}{cc}
N+1 & -1 \\
N & 1
\end{array}\right) \Gamma_{0}^{2}(N), & N \text { is even }
\end{array}\right. \text { - }
$$

Proof. First, we suppose that $N$ is even. Let's show that $\Gamma_{0}^{2}(N) \cup$ $\left(\begin{array}{cc}N+1 & -1 \\ N & 1\end{array}\right) \Gamma_{0}^{2}(N)=\Gamma_{0}(N)$. We have that $T:=\left(\begin{array}{cc}a & b \\ c N & d\end{array}\right) \in \Gamma_{0}(N)$ with $a d-$ $b c N=1$. Here, $a$ and $d$ are odd. If $b$ is even, $T$ would be an element of $\Gamma_{0}^{2}(N)$. We suppose that $b$ is odd. Hence, it can be written as $T=\left(\begin{array}{cc}N+1 & -1 \\ N & 1\end{array}\right)\left(\begin{array}{cc}x & y \\ c N & z\end{array}\right)$. Then, we have that $\left(\begin{array}{cc}1 & 1 \\ -N & N+1\end{array}\right)\left(\begin{array}{cc}a & b \\ c N & d\end{array}\right)=\left(\begin{array}{cc}x & y \\ c N & z\end{array}\right)$. Let's say that

$$
\underbrace{\left(\begin{array}{cc}
a+c N & b+d \\
-a N+c N(N+1) & -b N+d N(N+1)
\end{array}\right)}_{A}=\left(\begin{array}{cc}
x & y \\
c N & z
\end{array}\right) \text {. }
$$

As $b+d$ is even, $A \in \Gamma_{0}^{2}(N)$.

Now, let $N$ be odd. In this case, assume that $b$ and $c$ are even in $T$. Then $a$ and $d$ are odd. Hence, $T$ is an element of $\Gamma_{0}^{2}(N)$. Moreover, it can be written as $T=\left(\begin{array}{cc}1 & 0 \\ N & 1\end{array}\right)\left(\begin{array}{cc}x & y \\ c N & z\end{array}\right)$. As above, let's say that $\underbrace{\left(\begin{array}{cc}a & b \\ (c-a) N & d-b N\end{array}\right)}_{B}=$ $\left(\begin{array}{cc}x & y \\ c N & z\end{array}\right)$. Since $d-b N$ is even, $B \in \Gamma_{0}^{2}(N)$. In the case: $b$-even and $c$-odd, it is clear that $B \in \Gamma_{0}^{2}(N)$. If $a$ and $d$ are even in the equation $a d-b c N=1, B \in \Gamma_{0}^{2}(N)$ again. Finally if $a$ is odd and $d$ is even (or vice versa), the result is the same. Consequently, we obtain that $\left|\Gamma_{0}(N): \Gamma_{0}^{2}(N)\right|=2$.

Therefore, from the above constructed equivalence relation " $\approx$ ", we get $\Gamma^{2}$-invariant equivalence relation on $\widehat{\mathbb{Q}}$ by $\Gamma_{0}^{2}(N)$. It is clear that, by Lemma $2.3, \Gamma^{2}$ acts imprimitively on $\hat{\mathbb{Q}}$.

Let $v=\frac{r}{s}$ and $w=\frac{x}{y}$ be elements of $\hat{\mathbb{Q}}$. Because of the transitive action, we have that $v=g_{1}(\infty)$ and $w=g_{2}(\infty)$ for some elements $g_{1}, g_{2} \in \Gamma^{2}$ of the form 


$$
g_{1}:=\left(\begin{array}{cc}
r & * \\
s & *
\end{array}\right), \quad g_{2}:=\left(\begin{array}{cc}
x & * \\
y & *
\end{array}\right) .
$$

From the relation

$$
v \approx w \text { if and only if } g_{1}^{-1} g_{2} \in \Gamma_{0}^{2}(N),
$$

we get

$$
v \approx w \text { if and only if } r y-s x \equiv 0(\bmod N) .
$$

By our general discussion of imprimitivity, the number of blocks under $\approx$ is given by $\Psi(N)=\left|\Gamma^{2}: \Gamma_{0}^{2}(N)\right|$. So the block of $\infty$ is obtained as

$$
[\infty]:=\left\{\frac{x}{y} \in \hat{\mathbb{Q}} \mid y \equiv 0(\bmod N)\right\}
$$

2.4. Lemma. $\Psi(N)=N \prod_{p \mid N}\left(1+\frac{1}{p}\right)$ where the product is over the distinct primes $p$ dividing $N$.

Proof. To calculate $\Psi(N)$ we use two decomposition of the index $\left|\Gamma: \Gamma_{0}^{2}(N)\right|$ as the following

$$
\left|\Gamma: \Gamma^{2}\right|\left|\Gamma^{2}: \Gamma_{0}^{2}(N)\right|=\left|\Gamma: \Gamma_{0}(N)\right|\left|\Gamma_{0}(N): \Gamma_{0}^{2}(N)\right| .
$$

Here, $\left|\Gamma: \Gamma^{2}\right|=2$ and $\left|\Gamma: \Gamma_{0}(N)\right|=N \prod_{p \mid N}\left(1+\frac{1}{p}\right)$ are well-known by $[13,16]$ and $[16,17]$ respectively. We prove that the index of $\left|\Gamma_{0}(N): \Gamma_{0}^{2}(N)\right|$ is equal to 2 in Lemma 2.3. Writing these values in above equation, the result is obvious.

\section{Suborbital Graphs for $\Gamma^{2}$ on $\hat{\mathbb{Q}}$}

In[18], Sims introduced the idea of the suborbital graphs of a permutation group $G$ acting on a set $\Delta$, these are graphs with vertex-set $\Delta$, on which $G$ induces automorphisms. We summarise Sims'theory as follows:

Let $(G, \Delta)$ be transitive permutation group. Then $G$ acts on $\Delta \times \Delta$ by $g(\alpha, \beta)=$ $(g(\alpha), g(\beta))(g \in G, \alpha, \beta \in \Delta)$. The orbits of this action are called suborbitals of $G$. The orbit containing $(\alpha, \beta)$ is denoted by $O(\alpha, \beta)$. From $O(\alpha, \beta)$ we can form a suborbital graph $G(\alpha, \beta)$ : its vertices are the elements of $\Delta$, and there is a directed edge from $\gamma$ to $\delta$ if $(\gamma, \delta) \in O(\alpha, \beta)$. A directed edge from $\gamma$ to $\delta$ is denoted by $\gamma \rightarrow \delta$. If $(\gamma, \delta) \in O(\alpha, \beta)$, then we will say that there exists an edge $\gamma \rightarrow \delta$ in $G(\alpha, \beta)$. In this paper our calculation concerns $\Gamma^{2}$, so we can draw this edge as a hyperbolic geodesic in the upper half-plane $\mathbb{H}$, that is, as euclidean semi-circles or half-lines perpendicular to the real line.

The orbit $O(\beta, \alpha)$ is also a suborbital graph and it is either equal to or disjoint from $O(\alpha, \beta)$. In the latter case $G(\beta, \alpha)$ is just $G(\alpha, \beta)$ with the arrows reversed and we call, in this case, $G(\alpha, \beta)$ and $G(\beta, \alpha)$ paired suborbital graphs. In the former case $G(\alpha, \beta)=$ $G(\beta, \alpha)$ and the graph consists of pairs of oppositely directed edges; it is convenient to replace each such pair by a single undirected edge, so that we have an undirected graph which we call self paired.

3.1. Definition. By a directed circuit in a graph we mean a sequence $v_{1}, v_{2}, \ldots, v_{m}$ of different vertices such that $v_{1} \longrightarrow v_{2} \longrightarrow \ldots \longrightarrow v_{m} \longrightarrow v_{1}$, where $m \geq 3$.

If $m=3$, then the circuit, directed or not, is called a triangle.

If $m=2$, then we will say the configuration $v_{1} \longrightarrow v_{2} \longrightarrow v_{1}$ is self paired. 
A graph which contains no circuit is called a forest.

The above ideas are also described in a paper by Neumann [12] and in books by Tsuzuku [20] and by Biggs and White [4], the emphasis being on applications to finite groups. The reader is refereed to $[1,2,3,6,7,9,10,11]$ for some relevant previous work on suborbital graphs.

If $\alpha=\beta$, then $O(\alpha, \alpha)=\{(\gamma, \gamma) \mid \gamma \in \Delta\}$ is the diagonal of $\Delta \times \Delta$. The corresponding suborbital graph $G(\alpha, \alpha)$, called the trivial suborbital graph, is self-paired: it consists of a loop based at each vertex $\gamma \in \Delta$. We shall be mainly interested in the remaining non-trivial suborbital graphs.

Since $\Gamma^{2}$ acts transitively on $\hat{\mathbb{Q}}$, each suborbital contains a pair $(\infty, v)$ for some $v \in \hat{\mathbb{Q}}$; writing $v=\frac{u}{N},(u, N)=1$ and $N \geq 0$. We denote this suborbital by $O_{u, N}$ and the corresponding suborbital graph by $G_{u, N}$.

3.1. Graph $G_{u, N}$. If $v=\infty$, we would have the simplest suborbital graph, namely $G_{1,0}=G_{-1,0}$. Therefore, we can take $v \in \mathbb{Q}$. Let $v^{\prime}=\frac{u^{\prime}}{N^{\prime}} \in \mathbb{Q}$. The necessary and sufficient condition for $O(\infty, v)=O\left(\infty, v^{\prime}\right)$ is that $v$ and $v^{\prime}$ are in the same orbit of $\Gamma_{\infty}^{2}$. Since $\Gamma_{\infty}^{2}$ is generated by $z: v \rightarrow v+2$, then $z\left(\frac{u}{N}\right)=\frac{u+2 N}{N}=\frac{u^{\prime}}{N^{\prime}}$. Therefore, we have that $N=N^{\prime}$ and $u \equiv u^{\prime}(\bmod 2 N)$. Hence, $G_{u, N}=G_{u^{\prime}, N^{\prime}}$ if and only if $N=N^{\prime}$ and $u \equiv u^{\prime}(\bmod 2 N)$. Consequently, for a fixed $N$ there are $2 \varphi(N)$ distinct suborbital graphs $G_{u, N}$ where $\varphi(N)$ is Euler's phi function.

3.2. Theorem. $\frac{r}{s} \rightarrow \frac{x}{y} \in G_{u, N}$ if and only if

(i) If $r$ is even, then $x \equiv \pm$ ur $(\bmod N), y \equiv \pm u s(\bmod N), y \not \equiv \pm u s(\bmod 2 N)$ and $r y-s x=\mp N$.

(ii) If $s$ is even, then $x \equiv \pm u r(\bmod 2 N), y \equiv \pm u s(\bmod N)$ and $r y-s x=\mp N$.

(iii) If $r$ and $s$ are odd, then $x \equiv \pm$ ur $(\bmod N), y \equiv \pm u s(\bmod 2 N)$ and $r y-s x=$ $\mp N$.

Proof. (i) Let $r$ be even. By the transitivity of $\Gamma^{2}$, without loss of generality, we assume that $\frac{r}{s}<\frac{x}{y}$ where all letters are positive integers. Thus, we have that $r y-s x<0$. Since $\frac{r}{s} \rightarrow \frac{x}{y} \in G_{u, N}$, there exist some $T=\left(\begin{array}{ll}a & b \\ c & d\end{array}\right) \in \Gamma^{2}$ such that $T\left(\frac{1}{0}, \frac{u}{N}\right)=\left(\frac{r}{s}, \frac{x}{y}\right)$. As $r y-s x<0$, the multiplication of $\left(\begin{array}{ll}a & b \\ c & d\end{array}\right)\left(\begin{array}{ll}1 & u \\ 0 & N\end{array}\right)$ is equal to $\left(\begin{array}{cc}-r & x \\ -s & y\end{array}\right)$ or $\left(\begin{array}{cc}r & -x \\ s & -y\end{array}\right)$. If the first case is valid, we have that $a=-r, c=-s, a u+b N=x$ and $c u+d N=y$. That is, $x \equiv-u r(\bmod N)$ and $y \equiv-u s(\bmod N)$. Since $r$ is even, then $a$ is also even. To have $T \in \Gamma^{2}, d$ must be odd. From $-u s+d N=y$, we have that $y \not \equiv \pm u s(\bmod 2 N)$.

(ii) Suppose $s$ is even. In a similar way, we see that $b$ and $c$ must be even because $T\left(\frac{1}{0}\right)=\frac{-r}{-s}=\frac{a}{c}$. As in (i), we may assume that $\left(\begin{array}{cc}a & b \\ c & d\end{array}\right)\left(\begin{array}{ll}1 & u \\ 0 & N\end{array}\right)=\left(\begin{array}{ll}-r & x \\ -s & y\end{array}\right)$. Hence, we have that $a=-r, c=-s, a u+b N=x, c u+d N=y$ and $r y-s x=-N$. That is, $-u r+b N=x$ and $-u s+d N=y$. Since $b$ is even, we have that $x \equiv-u r(\bmod 2 N)$ and $y \equiv-u s(\bmod N)$.

(iii) Let $r$ and $s$ be odd. With similar argument, it can be seen that $d$ must be even. From the same matrix equation in (ii), we obtain that $x \equiv-u r(\bmod N)$ and $y \equiv-u s(\bmod 2 N)$. 
In the opposite direction, we shall prove (i) for minus sign. Suppose that $r$ is even, $x \equiv-u r(\bmod N), y \equiv-u s(\bmod N), y \not \equiv-u s(\bmod 2 N)$ and $r y-s x=-N$. In this case, there exist integers $b, d$ such that $x=-u r-b N, y=-u s-d N$. So, it is clear that $\left(\begin{array}{ll}-r & -b \\ -s & -d\end{array}\right) \in \Gamma^{2}$ which means $\frac{r}{s} \rightarrow \frac{x}{y} \in G_{u, N}$. Because $-N=r y-s x=$ $r(-u s-d N)-s(-u r-b N)$. This implies $-r d+s b=1$. As $r$ is even, $d$ must be even. Otherwise, it contradicts our hypothesis. With similar argument, we obtain the elements of $\Gamma^{2}$ which are $\left(\begin{array}{cc}-r & 2 b \\ -s & d\end{array}\right)$ and $\left(\begin{array}{cc}-r & -b \\ -s & -2 d\end{array}\right)$ for (ii) and (iii) respectively. .

3.3. Theorem. All suborbital graphs for $\Gamma^{2}$ on $\hat{\mathbb{Q}}$ are paired.

Proof. Because of the transitivity of $\Gamma^{2}$, it is sufficient to show that $G\left(\infty, \frac{u}{N}\right) \neq$ $G\left(\frac{u}{N}, \infty\right)$. It means that there is no $T \in \Gamma^{2}$ which sends the pair $\left(\infty, \frac{u}{N}\right)$ to the pair $\left(\frac{u}{N}, \infty\right)$. On the contrary, assume that $T(\infty)=\frac{u}{N}$ and $T\left(\frac{u}{N}\right)=\infty$. By comparing the determinants, we have that

$$
\left(\begin{array}{ll}
a & b \\
c & d
\end{array}\right)\left(\begin{array}{ll}
1 & u \\
0 & N
\end{array}\right)=\left(\begin{array}{cc}
-u & 1 \\
-N & 0
\end{array}\right) \text { or }\left(\begin{array}{ll}
a & b \\
c & d
\end{array}\right)\left(\begin{array}{ll}
1 & u \\
0 & N
\end{array}\right)=\left(\begin{array}{cc}
u & -1 \\
N & 0
\end{array}\right) .
$$

In the first case, we obtain $a=-u, c=-N, a u+b N=1$ and $c u+d N=0$. That is, $d=u$ and $u^{2}=-1+b N$. Taking $T=\left(\begin{array}{cc}-u & b \\ -N & u\end{array}\right)$ we see that the only case for $T$ to be an element of $\Gamma^{2}$ is that $N$ and $b$ must be even. Since $u^{2}=-1+b N$, then $u^{2} \equiv-1(\bmod b N)$. As $N$ and $b$ are even, $u^{2} \equiv-1(\bmod 4)$ which has no solution. For the second case, taking $T=\left(\begin{array}{cc}u & b \\ N & -u\end{array}\right)$, similar contradiction is obtained.

3.4. Corollary. There are no self-paired suborbital graphs for $\Gamma^{2}$ on $\hat{\mathbb{Q}}$.

In section 2 we introduced, for each integer $N$, a $\Gamma^{2}$-invariant equivalence relation $\widetilde{N}$ on $\hat{\mathbb{Q}}$, with $\frac{r}{s} \underset{N}{\approx} \frac{x}{y}$ if and only if $r y-s x \equiv 0(\bmod N)$. If $\frac{r}{s} \rightarrow \frac{x}{y}$ in $G_{u, N}$, then Theorem 3.2 implies that $r y-s x= \pm N$, so $\frac{r}{s} \underset{N}{\approx} \frac{x}{y}$. Thus, each connected component of $G_{u, N}$ lies in a single block for $\underset{N}{\approx}$, of which there are $\Psi(N)$, so we have:

3.5. Corollary. The graph $G_{u, N}$ is a disjoint union of $\Psi(N)$ subgraphs.

3.2. Subgraph $F_{u, N}$. We represent the subgraph of $G_{u, N}$ whose vertices form the block $[\infty]=\{x / y \in \hat{\mathbb{Q}} \mid y \equiv 0(\bmod N)\}$ by $F_{u, N}$.

3.6. Corollary. The graph $G_{u, N}$ consists of $\Psi(N)$ disjoint copies of $F_{u, N}$.

Proof. The vertices of each subgraph form a single block with respect to the $\Gamma^{2}$-invariant equivalence relation $\underset{N}{\approx}$ defined by $r y-s x \equiv 0(\bmod N)$. Therefore, if $x_{1} \rightarrow x_{2}$ is an edge in the subgraph $F_{u, N}, T\left(x_{1}\right) \rightarrow T\left(x_{2}\right)$ is also an edge in any other subgraph with $T \in \Gamma^{2}$ because of the transitivity of $\Gamma^{2}$ on $\hat{\mathbb{Q}}$.

Now, Theorem 3.2 immediately gives:

3.7. Theorem. $\frac{r}{s} \rightarrow \frac{x}{y} \in F_{u, N}$ if and only if

(i) If $r$ is even, then $x \equiv \pm u r(\bmod N), y \equiv \pm u s(\bmod N), y \not \equiv \pm u s(\bmod 2 N)$ and $r y-s x=\mp N$.

(ii) If $s$ is even, then $x \equiv \pm$ ur $(\bmod 2 N), y \equiv \pm u s(\bmod N)$ and $r y-s x=\mp N$.

(iii) If $r$ and $s$ are odd, then $x \equiv \pm$ ur $(\bmod N), y \equiv \pm u s(\bmod 2 N)$ and $r y-s x=$ $\mp N$. 
3.8. Theorem. $\Gamma_{0}^{2}(N)$ permutes the vertices and the edges of $F_{u, N}$ transitively.

Proof. Let $v, w$ be any vertices of $F_{u, N}$. Since $\Gamma^{2}$ acts on $\hat{\mathbb{Q}}$ transitively, there exist $T \in \Gamma^{2}$ such that $T(v)=w$. Taking $T=\left(\begin{array}{ll}a & b \\ c & d\end{array}\right), v=\frac{k_{1}}{l_{1} N}$ and $w=\frac{k_{2}}{l_{2} N}$ we see that

$N \mid c$. It means that $\Gamma_{0}^{2}(N)$ permutes the vertices of $F_{u, N}$.

Let $\frac{x_{1}}{y_{1} N} \stackrel{e_{1}}{\longrightarrow} b_{1}$ and $\frac{x_{2}}{y_{2} N} \stackrel{e_{2}}{\longrightarrow} b_{2}$ be any edges of $F_{u, N}$. We can give following diagram:

$$
\begin{aligned}
& \left(\frac{1}{0}, \frac{u}{N}\right) \stackrel{T_{2}}{\longrightarrow}\left(\frac{x_{2}}{y_{2} N}, b_{2}\right) \\
& \quad \downarrow T_{1} \nearrow_{T_{2} \circ T_{1}^{-1}} \\
& \left(\frac{x_{1}}{y_{1} N}, b_{1}\right)
\end{aligned}
$$

By this representation, we have $T_{1}=\left(\begin{array}{cc}x_{1} & * \\ y_{1} N & *\end{array}\right)$ and $T_{2}=\left(\begin{array}{cc}x_{2} & * \\ y_{2} N & *\end{array}\right)$. Since $T_{2} \circ T_{1}^{-1}$ has the form $\left(\begin{array}{cc}* & * \\ k N & *\end{array}\right)$ for some integer $k$, then $T:=T_{2} \circ T_{1}^{-1} \in \Gamma_{0}^{2}(N)$. It is clear that $T\left(\frac{x_{1}}{y_{1} N}\right)=\frac{x_{2}}{y_{2} N}$ and $T\left(b_{1}\right)=b_{2}$. Since $T$ is an element of a group of hyperbolic isometries of $\mathbb{H}$, geodesics are sent to geodesics under its action. So, $T$ transform the edges $e_{1}$ to $e_{2}$. Consequently, $\Gamma_{0}^{2}(N)$ permutes the edges of $F_{u, N}$.

3.9. Lemma. There is an isomorphism $F_{u, N} \longrightarrow F_{-u, N}$ given by $v \longrightarrow-v$.

Proof. It is clear that $v \longrightarrow-v$ is one-to-one and onto. Let's show that the structure is preserved. Here, it means that if $a \rightarrow b \in F_{u, N}$, then $-a \rightarrow-b \in F_{-u, N}$. Suppose that $\frac{r}{s} \rightarrow \frac{x}{y} \in F_{u, N}$ and $r$ is even. By Theorem 3.7(i), taking $\frac{r}{s}<\frac{x}{y}$, we have that $x \equiv-u r(\bmod N), y \equiv-u s(\bmod N), y \not \equiv-u s(\bmod 2 N)$ and $r y-s x=-N$. Since $\frac{r}{s}<\frac{x}{y}$, then $\frac{-r}{s}>\frac{-x}{y}$. Taking $-x \equiv(-u)(-r)(\bmod N), y \equiv(-u) s(\bmod N)$, $y \not \equiv(-u) s(\bmod 2 N)$ and $-r y+s x=N$, we have that $\frac{-r}{s} \rightarrow \frac{-x}{y} \in F_{u, N}$. For other conditions, the rest are similar.

3.10. Lemma. If $M \mid N$, then there is a homomorphism $F_{u, N} \longrightarrow F_{-u, M}$ given by $v \longrightarrow$ $-N v / M$.

Proof. We suppose that $\frac{r}{s N}, \frac{x}{y N}$ are adjacent vertices in $F_{u, N}$ and $\frac{r}{s N}<\frac{x}{y N}$ and that is written as $\frac{r}{s N} \stackrel{<}{\longrightarrow} \in \frac{x}{y N} \in F_{u, N}$. If $r$ is even, then $x \equiv-u r(\bmod N), y N \equiv$ $-u s N(\bmod N), y N \not \equiv-u s(\bmod 2 N)$ and $r y-s x=-1$. Since $M \mid N, x \equiv-u r(\bmod M)$, $y M \equiv-u s M(\bmod M), y M \not \equiv-u s(\bmod 2 M) . r y-s x=-1$ is also true for $M$. For other conditions, the rest are similar.

3.11. Theorem. $F_{u, N}$ contains directed triangles if and only if $u^{2} \mp u+1 \equiv 0(\bmod N)$.

Proof. Suppose that $F_{u, N}$ contains a directed triangle. Because of the transitive action, the form of directed triangle can be taken as $\infty \rightarrow \frac{u}{N} \stackrel{r}{\longrightarrow} \rightarrow \infty$ for some integer $r$. First, let $u$ be even. From the second edge, we have $u-r=-1$ and $r \equiv-u^{2}(\bmod N)$ by Theorem 3.2. So, we obtain $u^{2}+u+1 \equiv 0(\bmod N)$. Similarly, if $\frac{u}{N} \stackrel{>}{\longrightarrow}$, then we see that $u^{2}-u+1 \equiv 0(\bmod N)$. Now, $N$ is even. By applying Theorem 3.2 to the second edge, we have $u-r=-1$ and $r \equiv-u^{2}(\bmod 2 N)$, giving $u^{2}+u+1 \equiv 0(\bmod 2 N)$. It is impossible, because there is no solution for this equivalence. Finally, suppose that $u, N$ are odd. Again, from the second edge, we have $u-r=-1$ and $r \equiv-u^{2}(\bmod N)$, giving $u^{2}+u+1 \equiv 0(\bmod N)$. If $\frac{u}{N} \longrightarrow \frac{r}{N}$, it would be $u^{2}-u+1 \equiv 0(\bmod N)$. Combining all of the equivalences, we obtain $u^{2} \mp u+1 \equiv 0(\bmod N)$.

Conversely, if $u^{2} \mp u+1 \equiv 0(\bmod N)$, we see that either $u+1 \equiv-u^{2}(\bmod N)$ or $-u+1 \equiv-u^{2}(\bmod N)$. Theorem 3.2. implies that there is an edge $\frac{u}{N} \rightarrow \frac{u+1}{N}$ with 
$\frac{u}{N}<\frac{u+1}{N}$ in $F_{u, N}$ or $\frac{u}{N} \rightarrow \frac{u-1}{N}$ with $\frac{u}{N}>\frac{u+1}{N}$ in $F_{u, N}$. Consequently, there is a directed triangle $\infty \rightarrow \frac{u}{N} \rightarrow \frac{u \pm 1}{N} \rightarrow \infty$ in $F_{u, N}$.

Let us give some examples. For $u, N$-odd, $\frac{1}{0} \rightarrow \frac{3}{13} \rightarrow \frac{4}{13} \rightarrow \frac{1}{0}$ or $\frac{1}{13} \rightarrow \frac{10}{9 \cdot 13} \rightarrow$ $\frac{9}{8 \cdot 13} \rightarrow \frac{1}{13}$ is a directed triangle in $F_{3,13}$. For $u$-even and $N$-odd, $\frac{1}{0} \rightarrow \frac{2}{7} \rightarrow \frac{3}{7} \rightarrow \frac{1}{0}$ or $\frac{1}{7} \rightarrow \frac{5}{4 \cdot 7} \rightarrow \frac{4}{3 \cdot 7} \rightarrow \frac{1}{7}$ is a directed triangle in $F_{2,7}$. For $N$-even, we know that there is no triangle.

Observation. We know that there is no triangle in $F_{u, 2 N_{0}}$ for $N$-even by Theorem 3.11. Because of the relationships between elliptic elements with circuits, our expectation is that there is no elliptic element of order 3 of the form $\left(\begin{array}{cc}u & 2 b \\ 2 N_{0} & d\end{array}\right) \in \Gamma^{2}$. Indeed, being an elliptic element of order 3 , it is well-known that $u+d= \pm 1$. Taking determinant of $\left(\begin{array}{cc}1-d & 2 b \\ 2 N_{0} & d\end{array}\right)$, we have $d-d^{2}-4 b N_{0}=1$. It is clear that there is no solution for $d-d^{2} \equiv 1(\bmod 4)$.

On the other hand, we know that the suborbital graph for modular group is a forest if and only if it contains no triangles [2]. Using this fact, we can give the following result;

3.12. Corollary. The graph $G_{u, N}$ is a forest if and only if $u^{2} \pm u+1 \not \equiv 0(\bmod N)$.

3.3. Connectedness. In this last section, we examine the connectedness of $F_{u, N}$.

3.13. Definition. A subgraph $K$ of $G_{u, N}$ is called connected if any pair of its vertices can be joined by a path in $K$.

3.14. Theorem. The subgraphs $F_{0,1}$ and $F_{1,1}$ are connected.

Proof. Here, to see the situation better, we write the edge conditions for $F_{0,1}$ and $F_{1,1}$ by Theorem 3.2 explicitly.

Case $F_{0,1}: \frac{r}{s} \rightarrow \frac{x}{y} \in F_{0,1}$ if and only if

(i) If $r$-even, then $y$-odd and $r y-s x=\mp 1$.

(ii) If $s$-even, then $x$-even and $r y-s x=\mp 1$.

(iii) If $r, s$-odd, then $y$-even and $r y-s x=\mp 1$.

We will show that each vertex $\frac{a}{b}$ of $F_{0,1}$ can be joined to $\infty$ by a path in $F_{0,1}$. It is clear for $b=1$. Since $(a, b)=1$, we can write the equation $a d-b c=-1$ by Bezout's identity. For this pair $(c, d)$ satisfying the equation we claim that $\frac{a}{b}$ can be joined with $\frac{c}{d}$ by above edge condition.

Subcase1. Suppose $a$-even. By the equation we have that $b, c$ must be odd and there are two possibilities for $d$. If $d$-odd, then $\frac{a}{b} \stackrel{i}{\longrightarrow} \frac{c}{d}$ (means that we have $\frac{c}{d} \rightarrow \frac{a}{b}$ by (i)). If $d$-even, then $\frac{c}{d} \stackrel{i i}{\longrightarrow} \frac{a}{b}$.

Subcase2. Let $b$-even. By the equation we have that $a, d$ must be odd and there are two possibilities for $c$. If $c$-odd, then $\frac{c}{d} \stackrel{i i i}{\longrightarrow} \frac{a}{b}$. If $d$-even, then $\frac{a}{b} \stackrel{i i}{\longrightarrow} \frac{c}{d}$.

Subcase3. Assume that $a$-odd and $b$-odd. By the equation it is impossible that $c, d$ are odd or even at once, so there are two possibilities. If $c$-odd and $d$-even, then $\frac{a}{b} \stackrel{i i i}{\longrightarrow} \frac{c}{d}$. If $c$-even and $d$-odd, then $\frac{c}{d} \stackrel{i}{\longrightarrow} \frac{a}{b}$.

Consequently $F_{0,1}$ is connected.

Case $F_{1,1}: \frac{r}{s} \rightarrow \frac{x}{y} \in F_{1,1}$ if and only if

(i) If $r$-even, then $y$-even and $r y-s x=\mp 1$. 
(ii) If $s$-even, then $x$-odd and $r y-s x=\mp 1$.

(iii) If $r, s$-odd, then $y$-odd and $r y-s x=\mp 1$.

Taking a vertex $\frac{a}{b}$ in $F_{1,1}$, there exists the equation $a d-b c=-1$ by Bezout's identity. We shall show that $\frac{a}{b}$ is adjacent to vertex $\frac{c}{d}$ in $F_{1,1}$.

Subcase1. Suppose $a$-even. By the equation we have that $b, c$ must be odd and there are two possibilities for $d$. If $d$-odd, then $\frac{c}{d} \stackrel{i i i}{\longrightarrow} \frac{a}{b}$. If $d$-even, then $\frac{a}{b} \stackrel{i}{\longrightarrow} \frac{c}{d}$.

Subcase2. Let $b$-even. By the equation we have that $a, d$ must be odd and there are two possibilities for $c$. If $c$-odd, then $\frac{a}{b} \stackrel{i i}{\longrightarrow} \frac{c}{d}$. If $c$-even, then $\frac{c}{d} \stackrel{i}{\longrightarrow} \frac{a}{b}$.

Subcase3. Assume that $a$-odd and $b$-odd. By the equation it is impossible that $c, d$ are odd or even at once, so there are two possibilities. If $c$-odd and $d$-even, then $\frac{c}{d} \stackrel{i i}{\longrightarrow} \frac{a}{b}$. If $c$-even and $d$-odd, then $\frac{a}{b} \stackrel{i i i}{\longrightarrow} \frac{c}{d}$.

Consequently, $F_{1,1}$ is connected.

3.15. Theorem. The subgraphs $F_{1,2}$ and $F_{3,2}$ are connected.

Proof. We shall show that each vertex $v=\frac{a}{2 b}(b \geq 1)$ of $F_{1,2}$ is joined to $\infty$ by a path in $F_{1,2}$. Since the pattern is periodic with period 2 , we can show by induction on $b$. If $b=1$, then $v=\frac{a}{2}$ can be joined with $\infty$. If $a=1$, it is clear that $\frac{1}{0} \rightarrow \frac{1}{2}$. If $a=1$, then $\frac{3}{2} \rightarrow \frac{1}{0}$ because $1 \equiv-3(\bmod 4)$ and $3 \cdot 0-2 \cdot 1=-2$. If $a=5$, then $\frac{1}{0} \rightarrow \frac{5}{2}$. The same holds for the rest periodically. So we can assume that $b \geq 2$.

To complete the proof, we show that $v$ is adjacent to a vertex $w=\frac{a}{2 b_{1}}$ with $b_{1}<b$. It means that, $w$ is connected by a path to $\infty$, and hence so is $v$. As $(a, b)=1$, there exist integers $c, d$ such that $a d-b c=1$. For some $k \in \mathbb{Z}$, replacing $c$ and $d$ by $c+k a$ and $d+k b$, we can suppose $0<d<b$.

(i) If $c$ is odd, then $w=\frac{c}{2 d}$ can be joined with $\frac{a}{2 b}$. Indeed, $\frac{a}{2 b} \longrightarrow \frac{c}{2 d}$ gives that $a \cdot 2 d-c \cdot 2 b=2$ and $c \equiv a(\bmod 4)$. If $c \neq \equiv a(\bmod 4)$, taking $c \equiv-a(\bmod 4)$ we obtain $\frac{a}{2 b} \leftarrow \frac{c}{2 d}$ by $2 b c-2 a d=-2$. Hence, if $c$ is odd, $\frac{a}{2 b}$ is adjacent to $\frac{c}{2 d}$ in $F_{1,2}$.

(ii) If $c$ is even, then $a-c$ is odd. As $0<b-d<b$, we can take $w=\frac{a-c}{2(b-d)}$, adjacent to $\frac{a}{2 b}$ because $2(b c-c d)=-2$. Here, if $2 a-c \not \equiv 0(\bmod 4)$, then we have that $a-c \equiv a(\bmod 4)$ and $2(a d-b c)=2$.

Consequently, $F_{1,2}$ is connected. By the isomorphism $F_{1,2} \rightarrow F_{-1,2}=F_{3,2}, F_{3,2}$ is also connected.

3.16. Corollary. All graphs $F_{u, 2}$ are connected.

3.17. Corollary. The graph $G_{u, 2}$ has $2 \cdot \psi(2)=6$ connected components. Its blocks are $[\infty],[1],[0]$. The connected components of $[\infty]$ are $F_{1,2}$ and $F_{3,2}$.

3.18. Theorem. The subgraphs $F_{1,3}, F_{2,3}, F_{4,3}$ and $F_{5,3}$ are not connected.

Proof. It is sufficient to study with $F_{1,3}$ and $F_{2,3}$. Because there is an isomorphism from $F_{1,3}\left(F_{2,3}\right)$ to $F_{5,3}\left(F_{4,3}\right)$ respectively.

Case $F_{1,3}$ : If $F_{1,3}$ is connected, then each vertex $v=\frac{a}{3 b}$ would be joined to $\infty$. We shall show that no vertices of $F_{1,3}$ where $1<v<2$ are adjacent to $\infty$. Further, we assert that there is no such a vertex $v$ adjacent to vertices outside this interval. Of course, there is at least some vertex of $F_{1,3}$ in this strip. Suppose $\frac{2}{3} \leq \frac{c}{3 d}<1<\frac{a}{3 b}<2$. Then we have $\frac{c}{d}<3<\frac{a}{b}$. This is impossible because $c d-a d=-1$. Similarly, if $1<\frac{k}{3 l}<\frac{f}{3 e} \leq \frac{7}{3}$, then $\frac{k}{l}<4<\frac{f}{e}$ contradicts $k e-l f=-1$. It means that no vertices of $F_{1,3}$ between 1 and 2 are adjacent to $\infty$ and that $F_{1,3}$ is not connected. 


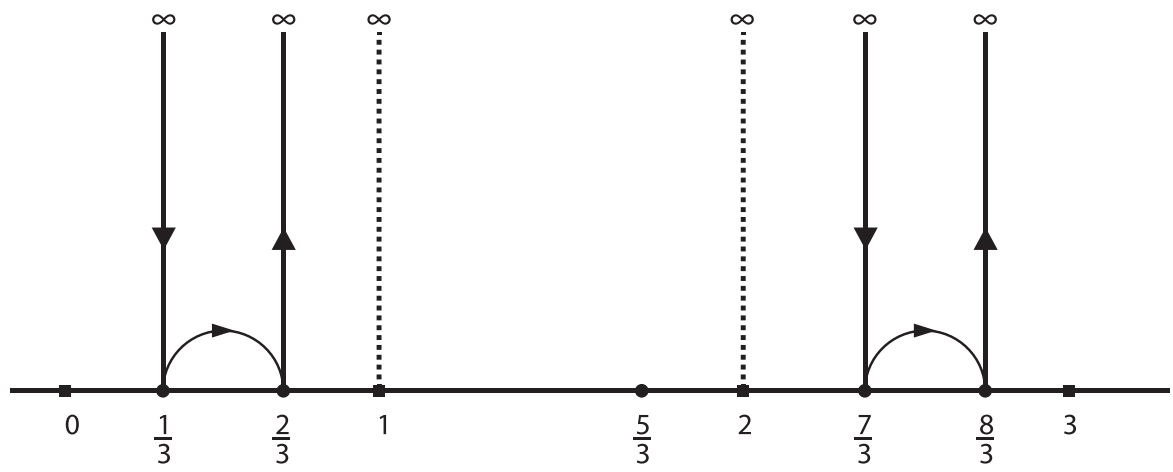

Figure 1. The subgraph $F_{1,3}$

Case $F_{2,3}$ : As above, let's show that no vertices of $F_{2,3}$ between $\frac{3}{2}$ and 2 are adjacent to vertices outside this interval. Suppose that $1 \leq \frac{x}{3 y}<\frac{3}{2}<\frac{a}{3 b}<2$ and $\frac{x}{3 y} \stackrel{<}{\longrightarrow} \frac{a}{3 b} \in F_{2,3}$. Then we have that $\frac{x}{y}<\frac{9}{2}<\frac{a}{b}$ and $x b-a y=-1$. By [7], we obtain that $x=4, y=1$, $a=5$ and $b=1$. But $\frac{4}{3} \rightarrow \frac{5}{3}$ is not in $F_{2,3}$. If $\frac{2}{3}<\frac{x}{3 y}<2<\frac{a}{3 b}<\frac{8}{3}$ and $\frac{x}{3 y} \stackrel{<}{\longrightarrow} \frac{a}{3 b} \in F_{2,3}$, then we would have $\frac{x}{y}<6<\frac{a}{b}$ and $x b-a y=-1$. It is impossible because of well-known Farey sequence. Consequently, $F_{2,3}$ is not connected.

3.19. Corollary. All graphs $F_{u, 3}$ are not connected.

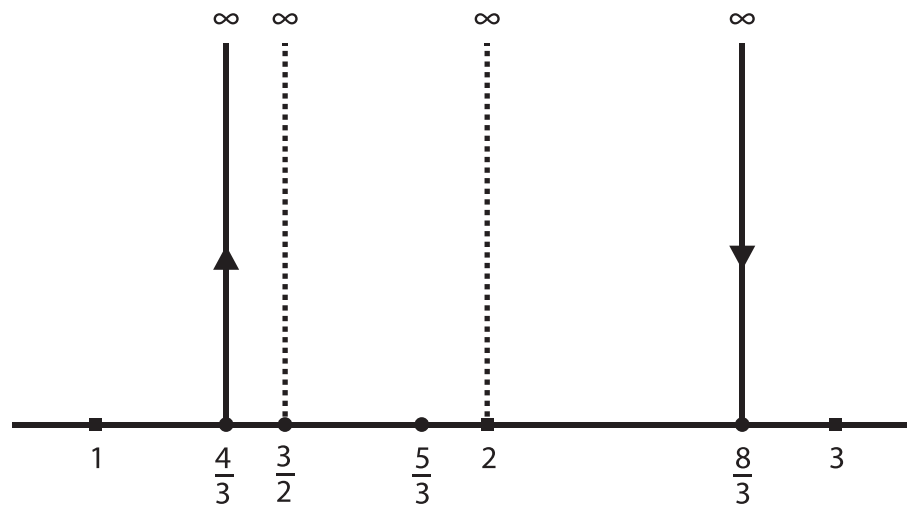

Figure 2. The subgraph $F_{2,3}$

3.20. Theorem. The subgraphs $F_{1,4}, F_{3,4}, F_{5,4}$ and $F_{7,4}$ are not connected.

Proof. As remarked in the proof of Theorem 3.18, it is sufficient to study with $F_{1,4}$ and $F_{3,4}$.

Case $F_{1,4}$ : We will show that no vertices in $F_{1,3}$ between $\frac{1}{2}$ and 1 are adjacent to vertices outside this interval. Suppose $\frac{1}{4} \leq \frac{a}{4 b}<\frac{1}{2}<\frac{x}{4 y}<1$. Then we have $\frac{a}{b}<2<\frac{x}{y}$. This is 
impossible because $a y-b x=-1$. Similarly, if $\frac{a}{4 b}<1<\frac{x}{4 y} \leq \frac{7}{4}$, then $\frac{a}{b}<4<\frac{x}{y}<7$ is a contradiction. So $F_{1,4}$ is not connected.

Case $F_{3,4}$ : As above, it is seen that no vertices of $F_{3,4}$ between 1 and 2 are adjacent to vertices outside this interval. Consequently, $F_{3,4}$ is not connected.

3.21. Theorem. The subgraph $F_{u, N}$ is connected if and only if $N \leq 2$.

Proof. If $F_{u, N}$ is connected, we know that $N \leq 4$ by [7]. For $N=3,4$, we proved that $F_{u, N}$ is not connected by Theorem 3.18 and 3.20. Conversely, if $N \leq 2$, the result immediately follows from Theorem 3.14 and 3.15 .

\section{References}

[1] Akbaş, M. and Başkan, T. Suborbital graphs for the normalizer of $\Gamma_{0}(N)$, Tr. J. of Mathematics, 20, 379-387, 1996.

[2] Akbaş, M. On suborbital graphs for the modular group, Bull. London Math. Soc., 33, 647$652,2001$.

[3] Beşenk, M. et al. Circuit lengths of graphs for the Picard group, J. Inequal. Appl., 2013:106, 8 pp., 2013.

[4] Bigg, N.L. and White, A.T. Permutation groups and combinatorial structures, London Mathematical Society Lecture Note Series, 33, CUP, Cambridge, 140 pp.,1979.

[5] Dixon, J. D. and Mortimer, B. Permutation Groups, Graduate Texts in Mathematics 163, Springer-Verlag, 1996.

[6] Güler, B.Ö. et al. Elliptic elements and circuits in suborbital graphs, Hacet. J. Math. Stat., 40 (2), 203-210, 2011.

[7] Jones, G.A., Singerman, D. and Wicks, K. The modular group and generalized Farey graphs, London Mathematical Society Lecture Note Series, 160, CUP, Cambridge, 316-338, 1996.

[8] Jones, G.A. and Jones, J.M. Elementary Number Theory, Springer Undergraduate Mathematics Series, Springer-Verlag, 1998.

[9] Kader, S., Güler, B. Ö. and Değer, A. H. Suborbital graphs for a special subgroup of the normalizer, IJST. Trans A., 34 (A4), 305-312, 2010.

[10] Kader, S. and Güler, B. Ö. On suborbital graphs for the extended Modular group $\hat{\Gamma}$, Graphs and Combinatorics, 29, no. 6, 1813-1825, 2013.

[11] Keskin, R. Suborbital graphs for the normalizer of $\Gamma_{0}(m)$, European J. Combin., 27, no. 2, 193-206, 2006.

[12] Neumann, P.M. Finite Permutation Groups, Edge-Coloured Graphs and Matrices, Topics in Group Theory and Computation, Ed. M. P. J. Curran, Academic Press, 1977.

[13] Newman, M. The Structure of some subgroups of the modular group, Illinois J. Math., 6, 480-487, 1962.

[14] Newman, M. Free subgroups and normal subgroups of the modular group, Illinois J. Math., 8, 262-265, 1964.

[15] Newman, M. Classification of normal subgroups of the modular group, Transactions of the American Math. Society, Vol.126, 2, 267-277, 1967.

[16] Rankin, R. A. Modular Forms and Functions, Cambridge University Press, 2008.

[17] Schoeneberg, B. Elliptic modular functions, Springer Verlag, 1974.

[18] Sims, C.C. Graphs and finite permutation groups, Math. Z., 95, 76-86, 1967.

[19] Singerman, D. Subgroups of Fuchsian groups and finite permutation groups, Bull. London Math. Soc., 2, 319-323, 1970.

[20] Tsuzuku, T. Finite Groups and Finite Geometries, Cambridge University Press, Cambridge, 1982. 